\section{Teledermatología asincrónica a través de WhatsApp : experiencia en la Pontificia Universidad Católica de Chile}

\author{
CATALINA HASBÚN ZEGPI ${ }^{1}$, MARÍA JESÚS ROJAS-LECHUGA ${ }^{\mathrm{a}}$, \\ JAVIER CONTADOR-GONZÁLEZ ${ }^{1, b}$, MAXIMILIANO CURI-TUMA², \\ MAURICIO SANDOVAL ${ }^{2}$
}

\section{Assessment of an asyncronic tele dermatology system for primary care physicians via social networking app WhatsApp}

Background: In Chile, there is little access to Dermatology outpatient consultations in the public health care system, which has favored the development of tele dermatology (TD). Aim: To assess satisfaction levels of primary care providers with a TD channel via the social networking app Whats $A p p^{\circledR}$ and the concordance between the diagnosis of general practitioners and dermatologists at Pontificia Universidad Católica de Chile. Material and Methods: An electronic survey was answered by the general practitioners who participate in the TD channel. In 417 cases, the diagnostic concordance between general practitioners and specialists was assessed. Results: The survey was answered by 84 practitioners. General satisfaction levels with the platform were over 95\%. Satisfaction levels with the response speed and the management suggested by the specialist were over $90 \%$. Over $80 \%$ of the practitioners read the consultation sent by dermatologists and considered that their dermatological knowledge improved. Diagnostic concordance between practitioners and specialists was 41\%. Conclusions: TD via a WhatsApp group linked to a university is a low cost and easy to implement intervention, generating high levels of satisfaction among general practitioners.

(Rev Med Chile 2020; 148: 1289-1294)

Key words: Dermatology; Primary Health Care; Telemedicine.

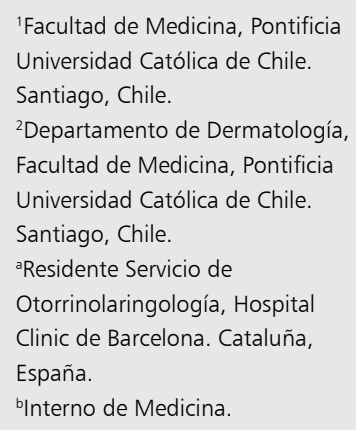

Trabajo no recibió financiamiento. Los autores declaran no tener conflictos de interés.

Recibido el 28 de julio de 2019 , aceptado el 24 de julio de 2020.

Correspondencia a:

Mauricio Sandoval Osses Departamento de Dermatología, Facultad de Medicina, Pontificia Universidad Católica de Chile. Avenida Vicuña Mackenna 4686, Macul. Santiago, Chile. msandoval2345@gmail.com
L a teledermatología (TD) es una de las disciplinas más desarrolladas dentro de la telemedicina, ya que el carácter visual de la especialidad favorece su uso por parte de médicos no especialistas para la orientación diagnóstica y/o terapéutica.

Existen dos modalidades: a) la de almacenamiento y retransmisión, en la cual la información del paciente es enviada como archivo digital y es evaluada en forma diferida y b) en tiempo real, que consiste en una videoconferencia entre el paciente o el médico general y el dermatólogo ${ }^{1}$. Chile es un excelente candidato para la implementación de plataformas de TD debido a su compleja geografía, la limitada oferta de consultas dermatológicas en el sistema público y la alta demanda en la Atención Primaria de Salud (APS) ${ }^{2}$.

Para permitir un acceso más fácil y rápido a interconsultas dermatológicas por parte de médicos que se desempeñan en APS, principalmente Médicos Generales de Zona (MGZ), en 2016 se creó un grupo de WhatsApp ${ }^{\circledR}$ de TD compuesto por médicos egresados de la Pontificia Universidad Católica de Chile (PUC), residentes de Dermatología y dermatólogos de dicha universidad, que hasta la fecha cuenta con más de 1.000 casos 
interconsultados y que tiene como moderador a uno de los autores (MSO). Se seleccionó la red social WhatsApp ${ }^{\varpi}$ debido a que es una herramienta gratuita y universal, que permite comunicarse de manera rápida.

El objetivo primario de este estudio es mostrar el nivel de satisfacción de los médicos generales con este recurso. Como objetivos secundarios, se evaluará la concordancia diagnóstica y se expondrán resultados con respecto a la satisfacción con la formación de pregrado en Dermatología que se realiza en la PUC.

\section{Material y Método}

Estudio descriptivo y transversal que evaluó diferentes grados de satisfacción por parte de los médicos egresados de la PUC en relación al uso del grupo de TD por Whatsapp ${ }^{\oplus}$, además de su opinión con respecto a la formación de pregrado de Dermatología, a través de una encuesta electrónica mediante la plataforma SurveyMonkey, previa firma de consentimiento informado.

La modalidad de uso consiste en el envío de casos clínicos en los que existe duda diagnóstica y/o terapéutica por parte de médicos generales. El grupo está administrado por dermatólogos y residentes de Dermatología de la PUC. Se permite el acceso a todo médico general egresado de la PUC que se desempeñe en la APS; el médico que desee integrarse solo debe solicitar el ingreso a través de un miembro del grupo. Los casos están estructurados de la siguiente manera: número de caso (correlativo a los casos enviados al grupo), médico a cargo, centro de salud, región, breve historia clínica, examen físico, exámenes de laboratorio pertinentes al caso y fotografías tomadas con el teléfono celular del médico. Esta información se envía a través de una serie de mensajes. En caso de que existan partes corporales reconocibles en las fotografías, éstas deben ser modificadas de acuerdo a la práctica habitual de publicación de fotografías clínicas para proteger la privacidad del paciente (por ejemplo, cuadros negros que cubran los ojos).

Existe un consenso entre los participantes del grupo que el médico a cargo cuenta con un consentimiento verbal por parte del paciente; en caso de menores de edad, éste es entregado por sus padres. El caso es enviado excluyendo cualquier dato privado para resguardar la confidencialidad del paciente. Posteriormente el caso es evaluado por especialistas del Departamento de Dermatología de la PUC (dermatólogos o residentes de dermatología supervisados), quienes realizan un diagnóstico y proponen un manejo incluyendo tratamiento y pertinencia de derivación al centro de referencia.

El grupo está organizado en base a un sistema de turnos semanales, en el cual existe un residente de Dermatología supervisado responsable de contestar los casos enviados, de manera de asegurar una respuesta relativamente rápida. En la mayoría de los casos esto ocurre dentro del día.

Se realizó una casuística de 442 diagnósticos enviados desde mayo de 2016 hasta junio de 2017, que se analizaron según el grupo de enfermedad.

Se excluyeron 25 casos, 20 en los que existía más de una posibilidad diagnóstica y 5 en los que no se pudo verificar el diagnóstico, recopilándose 417 casos en total en una planilla de Microsoft Excel $^{\oplus}$. Los datos se agruparon según diagnóstico específico dermatológico, para lo cual se usó de referencia el Atlas de Dermatología Clínica Fitzpatrick séptima edición y el consenso por parte del equipo de Dermatología. Además, con la misma base de datos se analizó el nivel de concordancia diagnóstica entre médicos generales y especialistas, para lo cual se utilizó el total de los casos (442). Se consideró concordante cuando al menos una hipótesis diagnóstica propuesta por el médico general era igual o similar al diagnóstico del dermatólogo o residente.

\section{Resultados (ver Anexo)}

\section{Caracterización demográfica}

El $80,95 \%$ de los 84 médicos encuestados eran MGZ, egresados principalmente el 2015 $(36,59 \%)$. Dentro de este grupo, el tipo de plaza con mayor reporte fue la C $(30,95 \%)$, seguido de la D $(21,43 \%)$. Las 5 regiones con más médicos encuestados fueron: Metropolitana (37,35\%), de Los Lagos (18,07\%), de La Araucanía (15,66\%), de Los Ríos $(8,43 \%)$ y del Libertador General Bernardo O’Higgins (7,23\%).

Más de un tercio de los encuestados (37,35\%) estimó que las consultas dermatológicas corresponden a $5 \%-10 \%$ de las consultas totales que realiza en su centro. El tiempo de espera aproximado para una interconsulta a Dermatología es entre 1 a 2 meses $(27,71 \%)$ y 2 a 6 meses $(27,71 \%)$. 
En cuanto a las alternativas de manejo de las patologías dermatológicas, se consultó sobre la disponibilidad de 9 medicamentos de uso común; los más disponibles fueron los antibióticos betalactámicos, los antimicóticos y los corticoides de baja potencia (Tabla 1 y pregunta 14 del Anexo). El 73,17\% dispone de cirugía menor.

\section{Uso del grupo de teledermatología y nivel de} satisfacción

De los encuestados, más de un tercio $(38,1 \%)$ utilizó el grupo de TD entre 3 y 5 veces. En cuanto a los grupos de enfermedad, el más representado

Tabla 1. Arsenal de medicamentos disponibles en los distintos centros de los médicos encuestados

\begin{tabular}{|lc|}
\hline Tipo de medicamento & Disponibilidad \\
\hline Amoxicilina/ácido clavulánico & $95,18 \%$ \\
\hline Cefadroxilo & $93,98 \%$ \\
\hline Fluconazol & $79,52 \%$ \\
\hline Corticoides de baja potencia & $73,49 \%$ \\
\hline Terbinafina & $65,06 \%$ \\
\hline Recetario Magistral & $57,83 \%$ \\
\hline Doxiciclina & $36,14 \%$ \\
\hline Corticoides de mediana potencia & $19,28 \%$ \\
\hline Permetrina 5\% & $18,07 \%$ \\
\hline
\end{tabular}

Tabla 2. Diagnósticos realizados a través de plataforma de TD

\begin{tabular}{|lc|}
\hline Diagnóstico & n de casos \\
\hline Inflamatorias & 210 \\
\hline Infecciosas & 91 \\
\hline Lesiones pigmentarias benignas & 45 \\
\hline Autoinmunes & 17 \\
\hline Tumores benignos & 12 \\
\hline Tumores malignos & 8 \\
\hline Enfermedades de los anexos & 6 \\
\hline Malformaciones vasculares y altera- & 3 \\
ciones del desarrollo & 2 \\
\hline Genodermatosis & 22 \\
\hline Otros & 1 \\
\hline No dermatológicos & \\
\hline
\end{tabular}

es el de las patologías inflamatorias con $50,4 \%$ de los diagnósticos (210), seguido de las infecciosas $(91,22 \%)$ y las lesiones pigmentarias benignas $(45,11 \%)$. Además hubo 8 casos en los que se sospecharon tumores malignos (Tabla 2 y Figura 1). La concordancia diagnóstica entre el médico general y el especialista fue de $40,72 \%$.

Con respecto al nivel de satisfacción con el grupo de TD, 73,49\% refiere estar muy satisfecho y $22,89 \%$ se encuentra satisfecho. Además, 95,24\% refiere estar muy satisfecho o satisfecho con la velocidad de respuesta de la teleconsulta. El $97,59 \%$ considera que el uso del grupo le ha facilitado el manejo y la derivación de pacientes dermatológicos, y 92,69\% refiere estar muy satisfecho o satisfecho con el manejo sugerido llevado a la realidad práctica-clínica.

\section{Conocimientos y formación de pregrado en Dermatología}

Desde el punto vista académico, 41,67\% refiere que ha ampliado de manera considerable su conocimiento dermatológico y $45,24 \%$ reporta que éste ha mejorado mediante el uso del grupo de TD. Además, 82,29\% de los médicos refiere que lee los casos de sus compañeros (42,86\% la mayoría de las veces, $26,19 \%$ algunas veces y $20,24 \%$ siempre). Con respecto a la formación de pregrado de Dermatología en la PUC, los encuestados la califican en $72,62 \%$ como buena, $14,29 \%$ como regular y

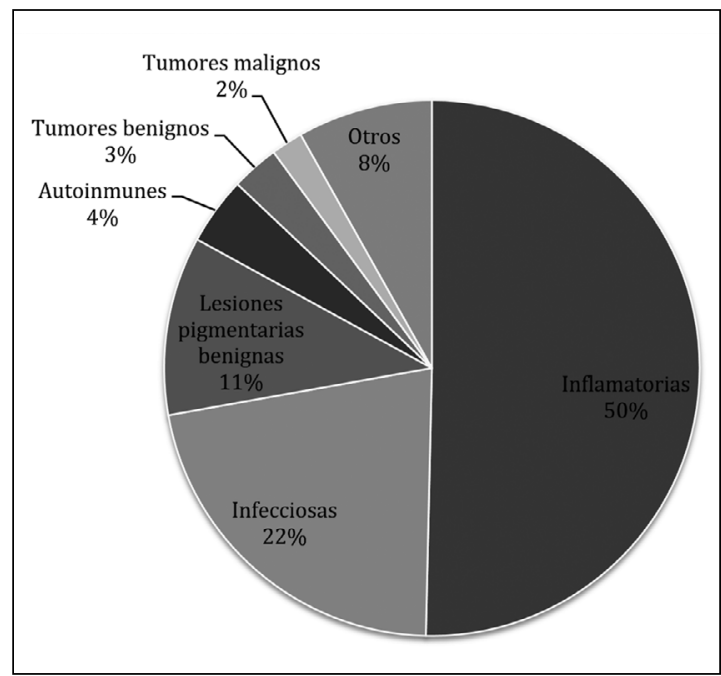

Figura 1. Distribución de diagnósticos realizados a través de TD según grupo de enfermedad. 
$10,71 \%$ como excelente. Sin embargo, $63,41 \%$ considera que hay conocimientos faltantes que debieron ser adquiridos durante su formación y $84,52 \%$ cree que es necesario adquirir conocimientos en cirugía dermatológica durante el pregrado. La mayoría $(67,86 \%)$ considera que el tiempo necesario de rotación en Dermatología durante el internado debería ser de 4 semanas, siendo la duración actual de dicho internado de 2 semanas.

\section{Discusión}

La telemedicina en general y particularmente la TD son disciplinas en constante desarrollo, ya que disminuyen la derivación a consultas de especialidad y permiten el manejo de patologías en la APS, aumentado la costo-efectividad del sistema de salud. Un estudio británico realizado en 2010 mostró que un servicio de TD por $e$-mail disminuyó las derivaciones en $68 \%$, además de aumentar el conocimiento de médicos generales y mejorar su confianza para realizar diagnósticos y manejar las patologías dermatológicas en su propia consulta ${ }^{3}$. En Latinoamérica destaca un estudio reciente realizado en Brasil, donde la implementación de una plataforma de TD evitó la derivación de 31.112 pacientes entre 2014 y 2016, quienes recibieron manejo y seguimiento en la APS ${ }^{4}$. Existen además experiencias reportadas en México ${ }^{5}$ y Colombia ${ }^{6}$.

La literatura parece estar de acuerdo en que las poblaciones más beneficiadas por la TD son aquellas con el menor acceso a la especialidad. En este contexto, aunque la mayoría de los diagnósticos dermatológicos requieren de una evaluación por especialista, los procedimientos tales como la toma de una biopsia de piel y el tratamiento y seguimiento de estos pacientes pueden ser realizados por un médico general ${ }^{7}$. Un estudio suizo realizado en un centro de TD en 2010 encontró que en la mitad de los casos los médicos generales podían completar el tratamiento de sus pacientes sin necesidad de una consulta de especialidad ${ }^{8}$.

Con respecto a las patologías que pueden ser diagnosticadas mediante TD y manejadas en la APS, un estudio español publicado en 2016 reveló que la mayor capacidad de resolución se alcanza en el grupo de enfermedades infecciosas, seguidas de las enfermedades inflamatorias, tumores benignos y lesiones pigmentadas benignas ${ }^{9}$. En el grupo de las lesiones malignas, otro estudio realizado en España determinó que la principal ventaja de la TD asincrónica es la mejoría en la calidad del tamizaje o cribado en la detección de lesiones malignas o sospechosas de malignidad ${ }^{10}$. Sin embargo, en el diagnóstico y manejo de dichas lesiones, las revisiones sistemáticas publicadas establecen que no existe suficiente evidencia que avale a la TD como una herramienta diagnóstica fiable, además de demostrar que existe un menor nivel de concordancia en el manejo de lesiones malignas cuando se compara la TD con la consulta presencial. Por lo tanto, la TD debería ser usada con precaución en lesiones pigmentadas sospechosas de malignidad ${ }^{11-14}$.

También se ha estudiado el uso de redes sociales para el desarrollo de plataformas de TD. Un estudio mexicano de 2018 en que se utilizó la red social Facebook ${ }^{\oplus}$ para el intercambio de casos clínicos entre un centro médico rural y un hospital urbano mostró que $75 \%$ de los pacientes pudo recibir un diagnóstico y tratamiento, sin necesidad de transportarse a otra ciudad para una consulta de especialidad ${ }^{15}$.

En Chile, existen escasos estudios de uso de TD. El primero fue publicado en 1999 e incorporó solo 5 casos provenientes de la Isla Juan Fernández, que fueron analizados por especialistas de un centro de salud privado en Santiago, y que reportó buenos resultados de evaluación del sistema por parte de médicos y pacientes ${ }^{16}$. Otro estudio publicado en 2011, que incluyó 172 teleconsultas de un centro de salud rural de la región del Maule analizadas por un teledermatólogo del Hospital Clínico de la Universidad de Chile, encontró una concordancia diagnóstica de $88,3 \%$, además de reportar altos niveles de satisfacción usuaria y de resolución de las teleconsultas ${ }^{17}$. Por último, un estudio de 2017 que analizó 1.764 teleconsultas derivadas desde el Servicio de Salud de Reloncaví a dermatólogos del Hospital El Pino en Santiago encontró una concordancia mayor a $60 \%{ }^{18}$.

En cuanto al uso de la plataforma de TD del Ministerio de Salud, un estudio publicado en 2015 evaluó la concordancia diagnóstica, terapéutica y de criterios de derivación entre una consulta dermatológica presencial y aquella realizada por un teledermatólogo en 5 comunas rurales dependientes del Servicio de Salud Metropolitano Occidente, encontrándose altos niveles de concordancia en todos los parámetros evaluados ${ }^{2}$. Un estudio más reciente realizado en zonas de aislamiento extremo 
del sur de nuestro país mostró buenos resultados en cuanto a concordancia diagnóstica entre el médico general y el teledermatólogo y tiempos de respuesta de la teleconsulta, además de reportar una disminución en la necesidad de derivación al centro de referencia ${ }^{19}$.

Este es el primer estudio chileno que evalúa un sistema de TD a través de teléfonos móviles ligado a una institución universitaria, y los resultados muestran que la distribución de diagnósticos según grupos de enfermedad es similar a la publicada en estudios internacionales ${ }^{9}$. Sin embargo, al realizar un análisis de la concordancia diagnóstica entre médicos generales y especialistas, se aprecia que ésta es inferior a 50\%. Los autores consideran que esto se debe a que, al ser un grupo docente en el que todos los médicos tienen acceso a los casos enviados, se va generando un aprendizaje, por lo que solo se envían casos nuevos y no se reenvían casos ya preguntados. Este sesgo impacta en el cálculo de la concordancia diagnóstica, ya que los casos van aumentando en complejidad, correspondiendo a patologías que son de manejo por el especialista.

Al encuestar a los médicos generales con respecto al funcionamiento del grupo de TD por WhatsApp ${ }^{\oplus}$, se observa que el nivel de satisfacción general supera 95\%, mientras que cuando se pregunta específicamente por la velocidad de respuesta de las teleconsultas y el manejo sugerido los niveles de satisfacción superan 90\%. También es una herramienta docente, ya que sobre $80 \%$ de los médicos encuestados considera que su conocimiento ha mejorado. Al ser un canal informal, permite que los miembros tengan acceso a los casos clínicos enviados por colegas, lo que se ve reflejado en $80 \%$ de los encuestados que refiere leer los casos enviados por otros médicos.

Cuando se evalúa la formación de pregrado en Dermatología en la PUC, se observa que sobre $70 \%$ la considera como buena; sin embargo, más de $60 \%$ cree que hay conocimientos faltantes que son necesarios para desempeñarse como médico general, principalmente en cirugía dermatológica. A pesar que no existen otros estudios nacionales que evalúen la docencia en Dermatología que se realiza en otras universidades, estas cifras podrían reflejar una falencia a nivel país en la formación médica, por lo que es aún más importante que existan plataformas de TD que permitan cubrir este déficit.
En conclusión, la implementación de un grupo de TD ligado a una institución universitaria a través de la red social WhatsApp es una intervención fácil de realizar, de bajo costo y efectiva en lograr mejorar el diagnóstico y manejo de pacientes con patologías dermatológicas atendidos por médicos generales, logrando altos niveles de satisfacción. El principal valor de esta herramienta es la docencia que se realiza, ya que todos los integrantes del grupo tienen acceso a los casos clínicos enviados y a las respuestas por parte de los especialistas. Sin embargo, es un medio inseguro para el tránsito de datos médicos, por lo que se quiere reportar como una experiencia y no para plantear su uso sistemático. Al ser un canal informal basado en redes sociales, es válido como una herramienta docente que se puede implementar en una institución universitaria, pero en ningún caso reemplaza a las plataformas tradicionales.

Agradecimientos: A todos los dermatólogos y residentes del Departamento de Dermatología de la Pontificia Universidad Católica de Chile que han participado en el grupo de teledermatología por WhatsApp ${ }^{\oplus}$, especialmente a: Dra. Renata Acle, Dra. Andrea Antúnez, Dra. Javiera Baeza, Dr. Leonardo Espinoza, Dr. Matías Gompertz, Dr. Agustín González, Dr. Rodrigo Meza, Dra. Lorena Montoya, Dra. Cynthia Moraga, Dra. Isabel Ogueta, Dra. Elena Ortiz, Dra. Loreto Paredes, Dr. Julio Parra, Dr. Cristián Pinto, Dr. Francisco Reyes, Dra. Josefina Rodríguez, Dr. Marco Silva, Dr. Marco Solís, Dra. María Paz Traipe, Dra. Elizabeth Valle, Dra. Verónica Vial y Dra. María José Zambrano.

\section{Referencias}

1. Trettel A, Eissing L, Augustin M. Telemedicine in dermatology: findings and experiences worldwide - a systematic literature review. J Eur Acad Dermatol Venereol 2018; 32 (2): 215-24.

2. Gatica JL, Bertoló S, Morales E, Espinoza M, Contreras C. Teledermatología en Chile, un aporte a la atención primaria de salud. Piel 2015; 30 (3): 148-54.

3. Crompton P, Motley R, Morris A. Teledermatology - the Cardiff experience. J Vis Commun Med 2010; 33 (4): 153-8.

4. Demo MLO, Marcon CEM. Comment on: 'A 14-year review of a UK teledermatology service: experience of over 40.000 teleconsultations'. Clin Exp Dermatol 2019. doi: 10.1111/ced.13998. [Epub ahead of print]. 
5. Chávez-López MG, Estrada-Chávez GE, Orozco-Figueroa M, Solís-Rivera A, Solchaga-Rosas J, Armendariz-Valle F, et al. Avances recientes en dermatología comunitaria 2016-2017. Teledermatología, un modelo de enseñanza y asistencia en atención primaria a la salud. Gac Med Mex 2018; 154 (Supp 2): S36-40.

6. Sáenz JP, Novoa MP, Correal D, Eapen BR. On using a mobile application to support teledermatology: a case study in an underprivileged area in Colombia. Int J Telemed Appl 2018: 1496941. doi: 10.1155/2018/1496941. eCollection 2018.

7. Muir J, Lucas L. Tele-dermatology in Australia. Stud Health Technol Inform 2008; 131: 245-53.

8. Maltagliati-Holzner P. Teledermatology in Switzerland: set-up for and examples of dermatological treatment from a telemedicine center. Hautarzt 2019; 70 (5): 32934 .

9. Batalla A, Suh-Oh HJ, Salgado-Boquete L, Abalde T, de la Torre C. Teledermatología. Capacidad para reducir consultas presenciales según el grupo de enfermedad. Piel 2016; 31 (3): 156-63.

10. Ferrer RT, Bezares AP, Mañes AL, Mas AV, Gutiérrez IT, Lladó CN, et al. Fiabilidad diagnóstica de una consulta de teledermatología asíncrona. Aten Primaria 2009; 41 (10): 552-7.

11. Bruce AF, Mallow JA, Theeke LA. The use of teledermoscopy in the accurate identification of cancerous skin lesions in the adult population: a systematic review. J Telemed Telecare 2018; 24 (2): 75-83.

12. Chuchu N, Dinnes J, Takwoingi Y, Matin RN, Bayliss SE, Davenport C, et al. Teledermatology for diagnosing skin cancer in adults. Cochrane Database Syst Rev 2018; 12: CD013193.

13. Warshaw EM, Hillman YJ, Greer NL, Hagel EM, MacDonald R, Rutks IR, et al. Teledermatology for diagnosis and management of skin conditions: a systematic review. J Am Acad Dermatol 2011; 64 (4): 759-72.

14. Warshaw EM, Lederle FA, Grill JP, Gravely AA, Bangerter AK, Fortier LA, et al. Accuracy of teledermatology for pigmented neoplasms. J Am Acad Dermatol 2009; 61 (5): 753-65.

15. García-Romero MT, Prado F, Domínguez-Cherit J, Hojyo-Tomomka MT, Arenas R. Teledermatology via a social networking site: a pilot study between a general hospital and a rural clinic. Telemed J E Health 2011; 17 (8): 652-5.

16. Lobos P. Teledermatología en las Islas Juan Fernández. Revista Médica Clínica Las Condes 1999; 10: 3.

17. Figueroa $A$, Barrios $X$, Radrigán $C$, Valdés $P$, Zemelman V. Aplicación de la teledermatología asincrónica en usuarios de la comuna de Yerbas Buenas, Región del Maule, Chile. Rev Chil Dermatol 2011; 27 (2): 170-6.

18. Fuenzalida H, Jimeno I, Toso S, Sepúlveda A, Loubies R. Teledermatología: impacto de una herramienta de gestión informática para zonas remotas de Chile. Piel 2017; 32 (5): 257-62.

19. González Coloma F, Sandoval Garcés M, Gedda Quiroga V, Bley Banda C. Teledermatología aplicada en zonas extremas: experiencia de 4 centros asistenciales chilenos en ámbito rural y aislamiento extremo. Actas Dermosifiliogr 2019. doi: 10.1016/j.ad.2019.01.009. [Epub ahead of print]. 